\title{
On the Effectiveness of the Eddy Straining Mechanism for the Maintenance of Blocking Flows
}

\author{
Miki ARAI and Hitoshi MUKOUGAWA ${ }^{1}$ \\ Graduate School of Environmental Earth Science, Hokkaido University, Sapporo, Japan
}

(Manuscript received 31 May 2001, in revised form 12 September 2001)

\begin{abstract}
In connection with the maintenance of blocking flows by the migrating synoptic disturbances, we examine the effectiveness of the eddy straining mechanism proposed by Shutts (1983) in an equivalent barotropic $\beta$-channel model. The model used here is identical to that in Haines and Marshall (1987) (hereafter referred to as HM) except with a channel twice as wide.

This model possesses two stationary solutions accompanying isolated structures in prescribed uniform westerlies when a vorticity forcing associated with the analytical modon solution is assumed: "blocking solution" similar to the modon solution and "zonal flow solution" characterized by dominant zonal flows. The infinitesimal transient eddies which mimic synoptic disturbances are generated by a wavemaker forcing located far upstream of the diffluence associated with the basic flow prescribed by stable stationary solutions. The effectiveness of the eddy straining mechanism is evaluated from the resemblance between the basic flow and the second-order flow induced by the time-averaged eddy potential vorticity (PV) flux divergence.

The distribution of the time-averaged PV divergence for the blocking solution in our model is the same as in HM in a sense that there is a PV north/south, divergence/convergence dipole upstream of the diffluence of the basic flow. However, the computed second-order flow has a quadruple structure which tends to shift the blocking dipole downstream instead of the dipole structure enforcing the blocking as shown in HM. On the other hand, the second-order flow tends to maintain a weak diffluence associated with the zonal solution. Thus, the effectiveness of the eddy straining mechanism depends on the basic flow. The second-order flow for the blocking solution is also drastically deformed by a negligible distortion of the PV divergence field associated with a small change in the property of imposed eddies. The effectiveness of the eddy straining mechanism depends also on the property of synoptic eddies.

Finally, this study suggests that the PV north/south, divergence/convergence dipole pattern does not necessarily maintain the blocking dipole. This remark is especially important for the observational study to assess the role of synoptic disturbances in the maintenance of blocking flows.
\end{abstract}

\section{Introduction}

The blocking events in the extratropical troposphere are characterized by a well known vortex pair with the anti-cyclone to the north

Corresponding author: Miki Arai, Graduate School of Environmental Earth Science, Hokkaido University, Sapporo 060-0810, Japan.

E-mail: arai@ees.hokudai.ac.jp

1 Present affiliation: Disaster Prevention Research Institute, Kyoto University, Uji, Kyoto.

(C) 2002, Meteorological Society of Japan and the cyclone to the south of the ambient jetstreams. However, the most important feature of the blocking in the context of the dynamic meteorology is its own unusual persistence in time beyond the typical time scale of synoptic eddies and of the Ekman surface friction (Hoskins et al. 1983).

One promising hypothesis regarding the maintenance of blocking against dissipation is the "eddy straining mechanism" proposed by Shutts (1983). The mutual interaction between synoptic transient eddies and stationary block- 
ing flows is the heart for this mechanism: when the migrating synoptic eddies advected by the zonal flow approach the diffluent straining field upstream of the blocking dipole, the eddies suffer an east/west compression and meridional extension, and are subsequently split meridionally. Associated with this strong deformation, a region of large eddy enstrophy is formed just upstream of the blocking dipole. By taking account of the balance in the time-averaged eddy enstrophy budget, the enhanced dissipation of the eddy enstrophy has to be canceled by downgradient (i.e., southward) eddy vorticity flux (see Eq. (2) in Shutts (1983)). This vorticity flux will in turn form vorticity divergence (convergence) to the north (south) of the axis of the ambient jetstreams. Since this dipole pattern of eddy forcing has the same polarity as the blocking vortex pair, we could anticipate that migrating synoptic eddies maintain blocking flows in a time-averaged sense against the surface friction.

By using an equivalent barotropic $\beta$-channel model, Shutts (1983) also exemplified the eddy straining mechanism by denoting similarity between the time-mean second-order circulation induced by transient eddies and the assumed simple diffluent basic flow prescribed by a stationary Rossby wave in the framework of a quasi-linear theory. Infinitesimal transient eddies which mimic synoptic-scale waves were generated by a simple wavemaker forcing located far upstream of the diffluent region.

Haines and Marshall (1987, hereafter referred to as HM) conducted numerical experiments to examine the eddy straining mechanism in almost the same framework as in Shutts (1983), but they adopted an isolated modon solution in a uniform westerly flow as the basic state. The modon solution with an isolated north-south dipole vortex is a stationary solution in an inviscid equivalent barotropic atmosphere and resembles the blocking flow pattern in the real atmosphere (McWilliams 1980). Because the obtained time-mean second-order circulation induced by imposed infinitesimal transient eddies is very similar to the basic flow, HM concluded that the eddy straining mechanism does work effectively for the maintenance of the basic flow comprised of the modon solution.

Although these two studies indicated the im- portant role of the eddy straining mechanism in the maintenance of the diffluent flow, the sensitivity of the effectiveness of this mechanism to the assumed basic flow and the property of eddies generated by the wavemaker was not examined. Moreover, there are some problems in the numerical procedure in HM: first, the channel width assumed in HM is too narrow to represent the modon solution which is analytically derived in an infinite $\beta$-plane. The streamfunction field of the modon solution in their channel model is noticeably distorted not only near the channel walls but also in the dipole vortex region. Second, the linear stability of the basic flow in their channel model was not confirmed. Thus, the obtained second-order flow induced by perturbations could be contaminated by unstable modes and does not necessarily represent the effect of the eddies propagating from upstream of the diffluent region.

Recently, Maeda et al. (2000) examined the eddy straining mechanism in a barotropic model on a sphere for realistic basic flows obtained from the observational data. They noted that the effectiveness of the eddy straining mechanism depends on the assumed basic flows and the imposed eddies. However, since the assumed basic flows are not any stationary solution, it is difficult to interpret the role of second-order induced flow by taking account of the time evolution of the basic flow itself. Moreover, the dependence of the effectiveness of the eddy straining mechanism on the characteristics of the imposed eddies was not revealed in detail.

In this study, we re-examine the effectiveness of the eddy straining mechanism for the maintenance of blocking flows, using an equivalent barotropic $\beta$-channel model identical to that in HM, except with a channel twice as wide. This model equation contains two stationary solutions when a vorticity forcing associated with the modon solution is assumed as indicated by Pierrehumbert and Malguzzi (1984) in a barotropic $f$-plane model: one solution closely resembles the modon solution; another solution accompanies dominant zonal flows. Thus, by adopting these stationary solutions under linearly stable conditions as the basic flow, it is able to argue in detail the effectiveness of the eddy straining mechanism without 
suffering from the interpretation of the secondorder flow. We will also indicate that the results in HM are harmfully affected by the channel walls in comparison with the results in our wide channel model, and the eddy straining mechanism is not so effective for the maintenance of the blocking flows. Moreover, the second-order flow is very sensitive to a fine structure of the eddy PV flux divergence field. This sensitivity is designated by changing several properties of the imposed eddies.

Since the analysis in this study is performed restricted within the framework of quasi-linear theory, the result obtained in this study is valid for infinitesimal transient eddies, and it is necessary to conduct fully nonlinear experiments for the next study. However, we think that the quasi-linear analysis in this study is helpful to improve our understandings on the time evolution of blocking flows in the nonlinear experiments.

\section{Model}

\subsection{Model description}

We use an equivalent barotropic quasigeostrophic potential vorticity equation on a $\beta$-channel with a vorticity source $\nabla^{2} \psi^{*}$, Ekman friction, and a scale-selective hyper-diffusion term:

$$
\frac{\partial q}{\partial t}+J(\psi, q)=\lambda\left(\nabla^{2} \psi^{*}-\nabla^{2} \psi\right)-v\left(\nabla^{2}\right)^{3} \psi .
$$

Here, $\nabla^{2}$ and $J$ denote the horizontal Laplacian and Jacobian operator, $\lambda$ and $v$ are the Ekman friction and the hyper-diffusion coefficient, $\psi$ the streamfunction, and $q$ the potential vorticity (PV) defined by

$$
q=f_{0}+\beta y+\nabla^{2} \psi-\gamma^{2} \psi .
$$

In (2), $f_{0}$ and $\beta$ are the Coriolis and beta parameters, respectively; $\gamma^{2} \equiv f_{0}^{2} / g H$, where $g$ is the acceleration of gravity and $H$ is the equivalent depth. Because we will examine isolated structures in a steady uniform zonal flow $U, \psi$ and $q$ are split into zonal and isolated eddy components;

$$
\left.\begin{array}{l}
\psi(x, y, t)=-U y+\tilde{\psi}(x, y, t), \\
q(x, y, t)=q_{\infty}(y)+\tilde{q}(x, y, t),
\end{array}\right\}
$$

where

$$
q_{\infty} \equiv f_{0}+\beta_{\infty} y=f_{0}+\beta y+\gamma_{\infty}^{2} U y,
$$

and

$$
\tilde{q}=\nabla^{2} \tilde{\psi}-\gamma^{2} \tilde{\psi}
$$

As shown in HM, for the existence of isolated stationary structures like modon solutions, which satisfy $\tilde{\psi} \rightarrow 0$ for $r \equiv\left(x^{2}+y^{2}\right)^{1 / 2} \rightarrow \infty$, the zonal flow $U$ has to be more barotropic than the isolated structure, i.e.,

$$
\gamma_{\infty}^{2}>\gamma^{2} \text {. }
$$

A cyclic boundary condition is imposed at $x=-L_{x} \pi$ and $x=L_{x} \pi$ :

$$
\tilde{\psi}(x, y, t)=\tilde{\psi}\left(x+2 \pi L_{x}, y, t\right) .
$$

Since two rigid walls of the channel are assumed at $y=-L_{y} \pi / 2$ and $y=L_{y} \pi / 2$, the following conditions

$$
\frac{\partial \tilde{\psi}}{\partial x}=0 \quad \text { and } \quad \int_{-L_{x} \pi}^{L_{x} \pi} \frac{\partial}{\partial t}\left(-\frac{\partial \tilde{\psi}}{\partial y}\right)=0,
$$

are applied at these walls.

Substituting (3) into (1), we obtain the following equation for isolated eddy components:

$$
\begin{aligned}
& \frac{\partial \tilde{q}}{\partial t}+J\left(\tilde{\psi}-y, \tilde{q}+\beta_{\infty} y\right) \\
& \quad=\lambda\left(\nabla^{2} \psi^{*}-\nabla^{2} \tilde{\psi}\right)-v \nabla^{6} \tilde{\psi},
\end{aligned}
$$

where all variables and constants are nondimensionalized: $t$ by $L_{y} / U, x$ and $y$ by $L_{y}, \tilde{\psi}$ and $\psi^{*}$ by $U L_{y}, \tilde{q}$ by $U / L_{y}, \beta_{\infty}$ by $U / L_{y}^{2}, \gamma$ by $L_{y}^{-2}, \lambda$ by $U / L_{y}$ and $v$ by $U / L_{y}^{3}$. We expand $\tilde{\psi}$ in the following truncated orthonormal functions which satisfy the boundary conditions (5) and (6):

$$
\left.\begin{array}{l}
F_{A_{m}}=\sqrt{2} \sin m y, \\
F_{K_{m}^{n}}=2 \cos m y \sin n \alpha x, \\
F_{L_{m}^{n}}=2 \cos m y \cos n \alpha x,
\end{array}\right\}
$$

where $m=1,2, \ldots, M, n=1,2, \ldots, N$, and $\alpha=$ $L_{y} / L_{x}$; the aspect ratio of this channel is given by $2 / \alpha$. Substituting the spectral expansion (8) into (7), a set of $M \times(2 N+1)$ ordinary differential equations for the expansion coefficients $x_{i}(t)$ is obtained.

\subsection{Modon solutions}

For an infinite $\beta$-plane, if we assume different linear functional relationships between $q$ and $\psi$ for the interior and the exterior regions of a circle with the center at the origin $(x, y)=(0,0)$ 
and the radius $r_{0}$, i.e.,

$$
\nabla^{2} \tilde{\psi}-\gamma^{2} \tilde{\psi}+\beta_{\infty} y=a(\tilde{\psi}-y),
$$

where

$$
a= \begin{cases}a_{1} & \text { for } r<r_{0}, \\ a_{2} & \text { for } r>r_{0},\end{cases}
$$

there is a set of isolated stationary solutions called "modon" for the inviscid case of $\lambda=v=0$ in (7). The assumption of isolated structures, i.e., $\tilde{\psi} \rightarrow 0$ for $r \rightarrow \infty$, means that

$$
a_{2}=-\beta_{\infty},
$$

from (9). Finally, if we impose the condition that the velocity is continuous at $r=r_{0}$ and the solution has a wavy structure for $r<r_{0}$, i.e., $a_{1}<-\gamma^{2}$, the modon solution,

$\psi= \begin{cases}\left\{r_{0} \frac{K_{1}(k r)}{K_{1}\left(k r_{0}\right)}-r\right\} \frac{y}{r}, & \text { for } r>r_{0}, \\ \frac{k^{2}}{\kappa^{2}}\left\{r-r_{0} \frac{J_{1}(k r)}{J_{1}\left(k r_{0}\right)}\right\} \frac{y}{r}, & \text { for } r<r_{0},\end{cases}$

is obtained, where $k^{2} \equiv \gamma^{2}-\beta_{\infty}, \quad \kappa^{2}=$ $-\left(\gamma^{2}+a_{1}\right), K_{1}$ and $J_{1}$ are the first order K- and J-Bessel function, respectively.

\subsection{Parameter values and truncation level}

In the numerical experiments, we use the following parameter values according to HM:

$$
\begin{aligned}
& U=13.8 \mathrm{~ms}^{-1}, \\
& \beta=1.6 \times 10^{-11} \mathrm{~m}^{-1} \mathrm{~s}^{-1}, \\
& \beta_{\infty}=1.2 \beta \mathrm{m}^{-1} \mathrm{~s}^{-1} \\
& L_{\rho} \equiv 1 / \gamma=845 \mathrm{~km}, \\
& a_{1}=-3.9 \times 10^{-12} \mathrm{~m}^{-2}, \\
& 2 \pi L_{x}=42,000 \mathrm{~km} .
\end{aligned}
$$

With these parameter values, Eq. (7) permits the existence of an analytical modon solution (12) with the radius of $r_{0}=2,430 \mathrm{~km}$. In order to examine the effect of the lateral boundaries on the model behavior, we analyze the model behaviors with two different meridional channel widths: narrow channel $\left(\pi L_{y}=10,500 \mathrm{~km}\right)$ corresponding to $\mathrm{HM}$ and wide channel $\left(\pi L_{y}=\right.$ $21,000 \mathrm{~km}$ ).

The truncation wave number adopted for the narrow channel is $M=21, N=42$ while $M=42, N=42$ for the wide channel. Thus, the horizontal resolution is the same for these two models and is fine enough to represent the dy- namics of the modon solution (see McWilliams et al. 1981). The hyper-diffusion coefficient $v$ is given to have an $e$-folding time of 1 day for the component of $M=21$ and $N=42$ for both models.

\subsection{Method for linearized experiments}

In order to assess the role of the migrating disturbances, which correspond to the synoptic eddies, in the maintenance of the basic flow, we examine the time-mean flow induced by infinitesimal disturbances generated by an imposed wavemaker forcing. For this analysis, we use the following perturbation expansion by a small parameter $\varepsilon$ :

$$
\left.\begin{array}{rl}
\tilde{\psi}(x, y, t)= & \psi_{0}(x, y)+\varepsilon \psi_{1}(x, y, t) \\
& +\varepsilon^{2} \psi_{2}(x, y, t)+O\left(\varepsilon^{3}\right), \\
\tilde{q}(x, y, t)= & q_{0}(x, y)+\varepsilon q_{1}(x, y, t) \\
& +\varepsilon^{2} q_{2}(x, y, t)+O\left(\varepsilon^{3}\right) .
\end{array}\right\}
$$

Substituting (14) into (7), and equating terms in the same order of $\varepsilon$ gives

$$
\begin{aligned}
\frac{\partial q_{0}}{\partial x} & +J\left(\psi_{0}, q_{0}\right)+\beta_{\infty} \frac{\partial \psi_{0}}{\partial x} \\
& =\lambda\left(\nabla^{2} \psi^{*}-\nabla^{2} \psi_{0}\right)-v \nabla^{6} \psi_{0}, \\
\left(\frac{\partial}{\partial t}+\right. & \left.\frac{\partial}{\partial x}\right) q_{1}+J\left(\psi_{1}, q_{0}\right)+J\left(\psi_{0}, q_{1}\right) \\
& +\beta_{\infty} \frac{\partial \psi_{1}}{\partial x}+\lambda \nabla^{2} \psi_{1}+v \nabla^{6} \psi_{1}=F_{1},
\end{aligned}
$$

and

$$
\begin{aligned}
\left(\frac{\partial}{\partial t}+\right. & \left.\frac{\partial}{\partial x}\right) q_{2}+J\left(\psi_{2}, q_{0}\right)+J\left(\psi_{0}, q_{2}\right)+\beta_{\infty} \frac{\partial \psi_{2}}{\partial x} \\
& +\lambda \nabla^{2} \psi_{2}+v \nabla^{6} \psi_{2}=-J\left(\psi_{1}, q_{1}\right) .
\end{aligned}
$$

Here, the vorticity forcing $\nabla^{2} \psi^{*}$ is assumed to have a magnitude of $O(1)$ such that the basic flow $\psi_{0}$ is a stationary solution of (15), and $F_{1}$ is an imposed wavemaker to generate migrating eddies $\psi_{1}$. We further prescribe $\nabla^{2} \psi^{*}$ by the vorticity distribution associated with the modon solution (12). Thus, we can expect that a stationary solution similar to the modon solution (12) is obtained as a basic flow $\psi_{0}$ in the channel model. We use the revised Marquadt method to obtain stationary solutions of (15) (see Mukougawa 1988).

The linear stability of the basic flow $\psi_{0}$ for infinitesimal disturbances $\psi_{1}$ is examined by 
using (16) without wavemaker forcing, i.e., $F_{1}=0$. By using the spectral expansion (8), Eq. (16) without $F_{1}$ is symbolically written as:

$$
\frac{d \boldsymbol{x}}{d t}+\mathrm{A} \boldsymbol{x}=0,
$$

where the vector $\boldsymbol{x}(t)$ consists of the expansion coefficients of eddy streamfunction $\psi_{1}$, and A is an $M(2 N+1) \times M(2 N+1)$ Jacobian matrix. The stability property of the basic state $\psi_{0}$ is examined by obtaining eigenvalues of the matrix A.

Now, the time-mean effect of infinitesimal disturbances generated by the wavemaker $F_{1}$ on the basic flow $\psi_{0}$ is assessed in the framework of a quasi-linear theory by the following procedures. First, we perform time integration of (16) for 50 days by using a 4th-order RungeKutta method with an imposed wavemaker $F_{1}$. According to Shutts (1983), the wavemaker is prescribed by

$$
\begin{aligned}
F_{1}= & \tilde{F}_{1} \sin \left\{\frac{\pi\left(x-x_{0}\right)}{\Delta x}\right\} \cos \left\{\frac{3 \pi\left(x-x_{0}-t\right)}{\Delta x}\right\} \\
& \times \sin \left\{\frac{\pi\left(y-y_{0}\right)}{\Delta y}\right\},
\end{aligned}
$$

for $\quad x_{0}<x<x_{1} ; \quad \Delta x=x_{1}-x_{0} ; \quad y_{0}<y<y_{1}$; $\Delta y=y_{1}-y_{0}$ where $\tilde{F}_{1}$ is a constant; otherwise $F_{1}=0$. Note that $F_{1}$ generates disturbances propagating eastward with the background uniform zonal wind speed (unit). The rectangular wavemaker region defined by $x_{0}, x_{1}, y_{0}$ and $y_{1}$ is located well upstream of the model origin $(x, y)=(0,0)$ where the diffluence associated with the basic flow exists.

Second, the last 45 days of the integration period, which are quite long compared with the characteristic period of synoptic eddies, are used to compute the time-mean eddy PV flux divergence $\overline{\boldsymbol{J}\left(\psi_{1}, q_{1}\right)}$, where the overbar means the time-average. Finally, we obtain the induced time-mean second-order flow $\overline{\psi_{2}}$ by solving the time-averaged equation of (17):

$$
\begin{aligned}
\frac{\partial \overline{q_{2}}}{\partial x} & +J\left(\overline{\psi_{2}}, q_{0}\right)+J\left(\psi_{0}, \overline{q_{2}}\right)+\beta_{\infty} \frac{\partial \overline{\psi_{2}}}{\partial x} \\
& +\lambda \nabla^{2} \overline{\psi_{2}}+v \nabla^{6} \overline{\psi_{2}}=-\overline{J\left(\psi_{1}, q_{1}\right)} .
\end{aligned}
$$

Note that the eddy PV flux divergence $\nabla \cdot\left(\overline{\boldsymbol{v}_{1} q_{1}}\right)$, which is also expressed as $\overline{J\left(\psi_{1}, q_{1}\right)}$ by using the wind vector $\boldsymbol{v}_{\mathbf{1}}=\left(-\partial \psi_{1} / \partial y, \partial \psi_{1} / \partial x\right)$, acts as the forcing term for $\psi_{2}$. From the similarity of the spatial structure of $\overline{\psi_{2}}$ to the basic flows, we could infer a tendency of the time evolution of the basic flow against the dissipative process due to an ensemble effect of imposed transient eddies.

The steady response of the second-order flow $\psi_{2}$ due to $-\overline{J\left(\psi_{1}, q_{1}\right)}$ in (20) is also examined by the singular value decomposition (SVD) analysis (see Navarra 1993; Itoh and Kimoto 1999; Maeda et al. 2000). The analysis is based on the following symbolically written equation (20):

$$
\mathrm{A} \boldsymbol{y}=\boldsymbol{f},
$$

where the vectors $\boldsymbol{y}$ and $\boldsymbol{f}$ represent the spectral expansion coefficients of $\overline{\psi_{2}}$ and $-\overline{J\left(\psi_{1}, q_{1}\right)}$, respectively; the matrix $A$ in (21) is the same that in (18). Then, the SVD of the matrix A gives the expansion of the induced second-order flow $\boldsymbol{y}$ in terms of the right singular vectors $\xi_{i}(i=1,2, \ldots, M(2 N+1))$ :

$$
\boldsymbol{y}=\sum_{i=1}^{M(2 N+1)} \frac{\left\langle\boldsymbol{f} \cdot \zeta_{i}\right\rangle}{\sigma_{i}} \xi_{i}
$$

where $\langle\cdot\rangle$ indicates the inner-product, $\sigma_{i}$ $\left(0 \leq \sigma_{1} \leq \sigma_{2} \ldots\right)$ and $\zeta_{i}$ are the $i$-th singular value and the $i$-th left singular vector. The singular values satisfy $\mathrm{AA}^{T} \zeta_{i}=\sigma_{i}^{2} \zeta_{i}$ or $\mathrm{A}^{T} \mathrm{~A} \xi_{i}=$ $\sigma_{i}^{2} \xi_{i}$, where the superscript $T$ means the transpose. Sets of vectors $\left\{\xi_{i}\right\}$ and $\left\{\zeta_{i}\right\}$ construct orthonormal basis, respectively.

From (22), we find that the flow pattern associated with $\xi_{j}$ will dominates the $\overline{\psi_{2}}$ field if the coefficient $\left\langle\boldsymbol{f} \cdot \zeta_{j}\right\rangle / \sigma_{j}$ of this mode $j$ is larger than the amplitude of the other modes $i$ for $i \neq j$. In particular, if the smallest singular value $\sigma_{1}$ is much smaller than the others while the projection of the forcing $\boldsymbol{f}$ onto each left singular vector $\zeta_{i},\left\langle\boldsymbol{f} \cdot \zeta_{i}\right\rangle$, has a comparable magnitude among all modes, the second-order flow $\overline{\psi_{2}}$ becomes very similar to $\xi_{1}$; this is expected when the basic flow $\psi_{0}$ satisfies near-resonant condition, i.e., $\sigma_{1} \approx 0$. For near-resonant basic flows, detailed structures of the forcing $f$ are irrelevant to determine the spatial structure of $\overline{\psi_{2}}$ field except for the sign of $\left\langle\boldsymbol{f} \cdot \zeta_{\mathbf{1}}\right\rangle$ which controls the polarity of $\overline{\psi_{2}}$.

\section{Narrow channel model}

In this section, we describe the results in the narrow channel model with lateral width $\pi L_{y}=$ $10,500 \mathrm{~km}$ which is the same that in HM. 


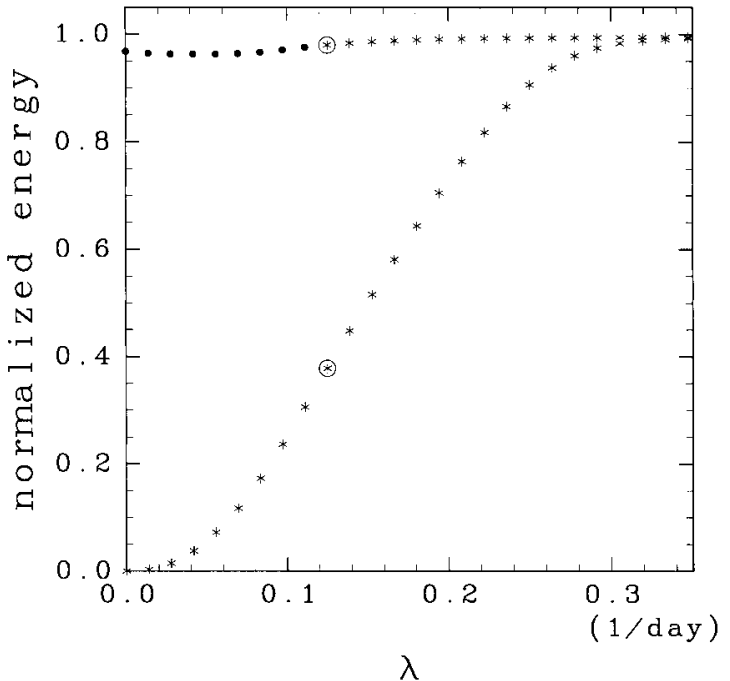

Fig. 1. Bifurcation diagram of steady solutions in the narrow channel model. The abscissa is the Ekman friction coefficient $\lambda\left(\right.$ day $\left.^{-1}\right)$ and the ordinate is the kinetic energy of steady solutions normalized by that of analytic modon solution of (12). The basic zonal flow is excluded in computing the kinetic energy. Linearly stable solutions are denoted by asterisks and unstable solutions by solid circles.

\subsection{Multiple stationary solutions}

We numerically obtain stationary solutions of (15) by assuming two initial guesses: one is the modon solution of (12) and the other is $\psi_{0}=0$. Obtained branches of stationary solutions are shown in Fig. 1 where the Ekman friction coefficient $\lambda$ is used as a bifurcation parameter and the ordinate denotes the kinetic energy of stationary solutions $\psi_{0}$ normalized by that of the analytic modon solution of (12). In this parameter range, the multiplicity of stationary solutions is clearly observed. Since the flow pattern closely resembles the analytical modon solution, the upper branch solutions (Fig. 2a) will be referred to as "blocking solution". On the other hand, the solutions belonging to the lower branch (Fig. 2b) have relatively weak zonally asymmetric components, and uniform zonal flow components dominate in the flow pattern. Thus, we will call this solution "zonal flow solution". With the increase of $\lambda$, the lower branch approaches the upper branch very

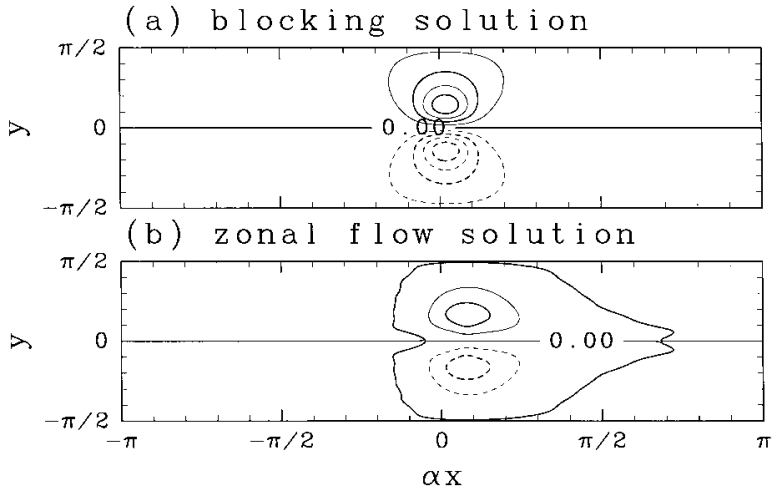

Fig. 2. Streamfunction of the steady solutions $\psi_{0}$ at $\lambda=0.13$ day $^{-1}$ denoted by open circles in Fig. 1. Contour interval is 0.2. Negative values are contoured by dashed lines. (a) Blocking solution. (b) Zonal flow solution.

closely, but a bifurcation point does not exist in this parameter range. The existence of similar multiple equilibria of isolated solutions was reported in Pierrehumbert and Malguzzi (1984) for an $f$-plane barotropic model, and also in Yamagata et al. (1990) for a $\beta$-plane equivalent barotropic model.

The linear stability property of stationary solutions is also depicted by two different symbols in Fig. 1. The blocking solution becomes unstable for $\lambda<0.13$ day $^{-1}$, while the zonal flow solution is stable in this parameter range. The growth rate of the most unstable mode for the blocking solution becomes larger with the decrease of $\lambda$ as shown in Fig. 3a: when $\lambda=0$, the growth rate attains the maximum value of 0.15 day $^{-1}$; this unstable mode is stationary with zero frequency.

We also confirm the instability of the analytical modon solution $(\lambda=v=0)$ in a higher horizontal resolution model with $M=42$ in (8). The numerically obtained fastest growing mode (Fig. 3b) for the analytical modon solution has a large amplitude in the center of the channel. The flow pattern of this unstable mode is similar to that obtained by the time integration from the initial condition composed of the westward-propagating modon solution as shown in Nycander (1992), which also gave a theoretical proof on the linear instability of the modon solution. 


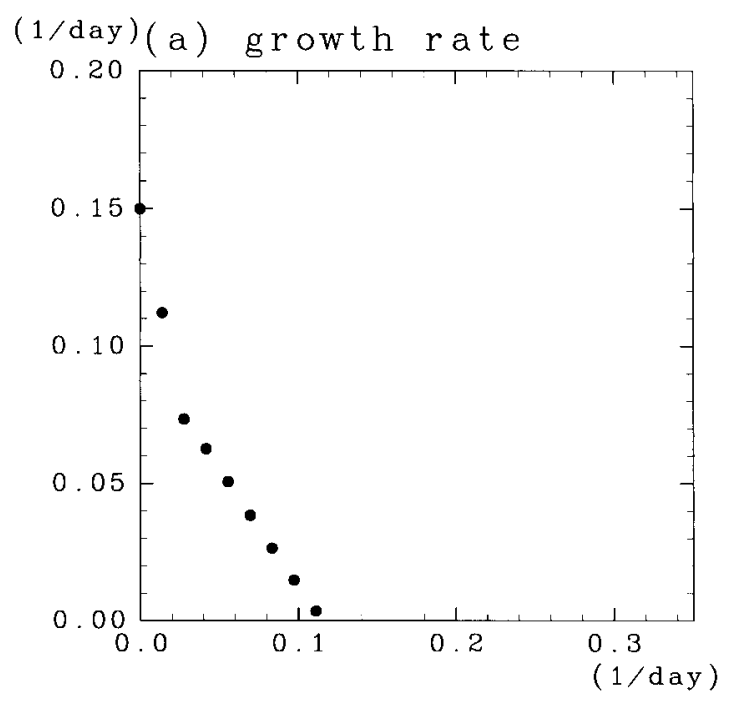

$\lambda$

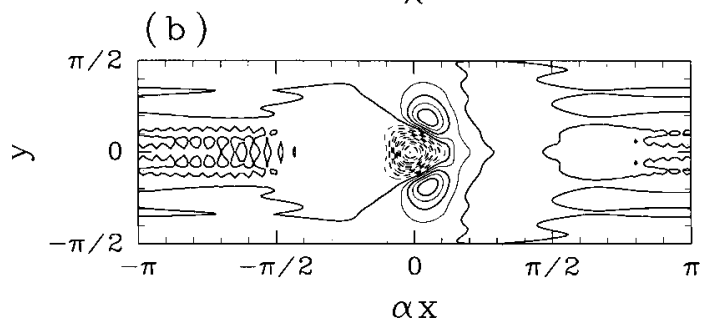

Fig. 3. (a) Dependence of the growth rate of the most unstable mode for the blocking solution on the Ekman friction coefficient $\lambda$. (b) The eigenvector of the most unstable mode for the analytical modon solution (12). This is a stationary mode with the growth rate of 0.15 day $^{-1}$. Negative values are contoured by dashed lines.

\subsection{Second-order flow}

Now, we will examine the second-order induced flow $\overline{\psi_{2}}$ associated with transient perturbations forced by a prescribed wavemaker of (19). When the basic flow is unstable to infinitesimal perturbations by assuming a small value of $\lambda$, it is difficult to assess the effect of imposed perturbations on the basic flow because the obtained induced flow will be contaminated by growing unstable modes. On the other hand, if we assume a large Ekman friction coefficient $\lambda$ to assure the stability of the basic flow, the transient eddies forced far upstream of the diffluent region of the basic flow will fade away before reaching the diffluence and producing significant vorticity flux. Thus,

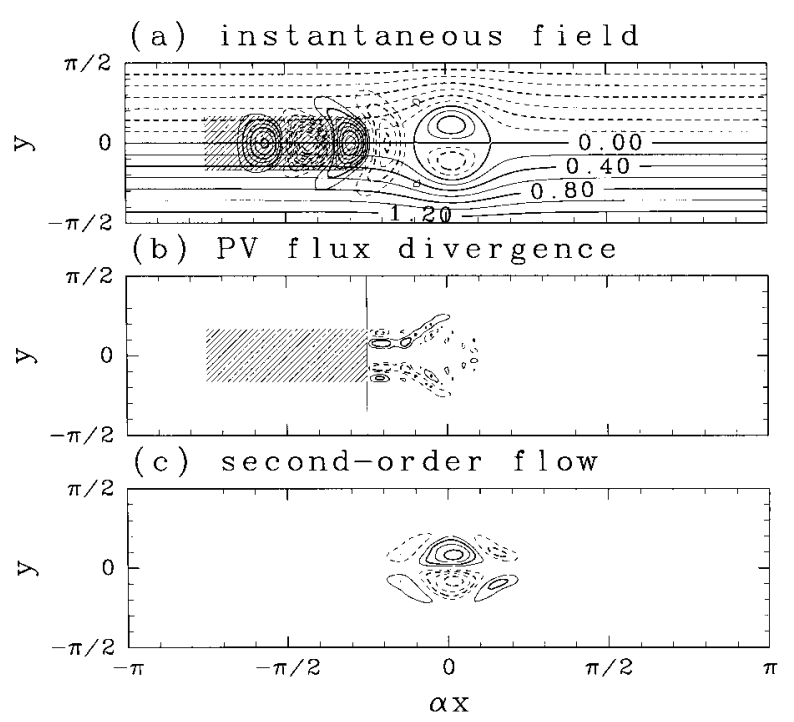

Fig. 4. Transient eddy forcing for the blocking basic flow in the narrow channel. Negative values are contoured by dashed lines. Zero contours are omitted. (a) Instantaneous streamfunction field of the disturbances $\psi_{1}$ at day 30 with the basic flow $\psi_{0}-U y$. Contour interval is $2.0 \times 10^{-4}$. The shaded rectangle denotes the eddy forcing region. (b) Timeaveraged eddy $\mathrm{PV}$ flux divergence $\nabla \cdot \overline{\left(\boldsymbol{v}_{1} q_{1}\right)}$ during 45 days. Contour interval is $1.0 \times 10^{-5}$. (c) Second-order flow $\overline{\psi_{2}}$ induced by the time-average eddy PV flux divergence downstream of the solid vertical line in (b). Contour interval is $1.0 \times 10^{-5}$.

we set $\lambda=0.12 \mathrm{day}^{-1}$ (denoted by open circles in Fig. 1) at which the blocking solution is marginally stable.

First, we consider the effect of perturbations on the blocking solution. Figure 4a shows an instantaneous field of the perturbation streamfunction $\psi_{1}$ at day 30 superimposed on the basic flow $\psi_{0}$. The hatched rectangular area denotes the wavemaker region given by (19), where we set $\alpha x_{0}=-3 \pi / 4, \alpha x_{1}=-\pi / 4, y_{0}=$ $-\pi / 6, y_{1}=\pi / 6$. The zonal wavelength and the period of the eddies forced by this wavemaker are $7,000 \mathrm{~km}$ and 5.8 days. The meridional wavelength of this perturbation is the same as the zonal wavelength i.e., the imposed eddy is isotropic. Approaching the diffluent region, perturbations expand in the lateral direction 
and shrink in the zonal direction. We also see a phase tilt associated with the differential advection by the basic flow $\psi_{0}$ in the upstream of the diffluence. The time-averaged PV flux divergence $\overline{J\left(\psi_{1}, q_{1}\right)}$ in (20) associated with the perturbations is shown in Fig. $4 \mathrm{~b}$ where the wavemaker region is masked. In the upstream of the diffluence, the horizontal distribution of eddy PV flux divergence is anti-symmetric with respect to the center of the channel $y=0$. There is enhanced divergence to the north of $y=0$ and convergence to the south in just upstream of the diffluence.

Second-order induced flow $\overline{\psi_{2}}$ forced by the eddy PV flux divergence in the downstream region of $x_{1}$ in Fig. $4 \mathrm{~b}$ is shown in Fig. 4c. The flow pattern is characterized by a north/south, anti-cyclonic/cyclonic vortex dipole, and is almost identical to that obtained in HM. Since the second-order flow has a similar horizontal structure to the basic flow and the polarity is also the same, we can anticipate that the transient disturbances have a tendency to enforce the diffluence of the basic blocking flow and the eddy straining mechanism does work in the narrow channel.

In order to investigate the sensitivity of the second-order flow $\overline{\psi_{2}}$ to the characteristic of the imposed forcing, we proceed the singular value decomposition analysis for the matrix $A$ in (18). Figure 5a indicates that the smallest singular value $\sigma_{1}$ is almost two orders of magnitude smaller than the second smallest one. Moreover, the right singular vector $\xi_{1}$ associated with $\sigma_{1}$ has a similar horizontal pattern to the second-order flow $\overline{\psi_{2}}$ in Fig. 4c. On the other hand, the corresponding inner-product $\left\langle\zeta_{\mathbf{1}} \cdot \boldsymbol{f}\right\rangle$ has a comparable magnitude with $\left\langle\zeta_{i} \cdot \boldsymbol{f}\right\rangle$ for $i \neq 1$ (not shown). Thus, in this narrow channel, we find that the basic flow is in a near-resonant condition, and the induced second-order flow is irrelevant to the characteristic of the eddy forcing except for the polarity of the dipole structure. We also confirm that the results for the blocking basic flow do not depend on the Ekman friction $\lambda$ when the basic flow is stable.

We have also performed the analysis on the second-order flow for the zonal flow solution but refrain from describing the results here because the obtained results are identical to those in the wide channel model documented in the next section.
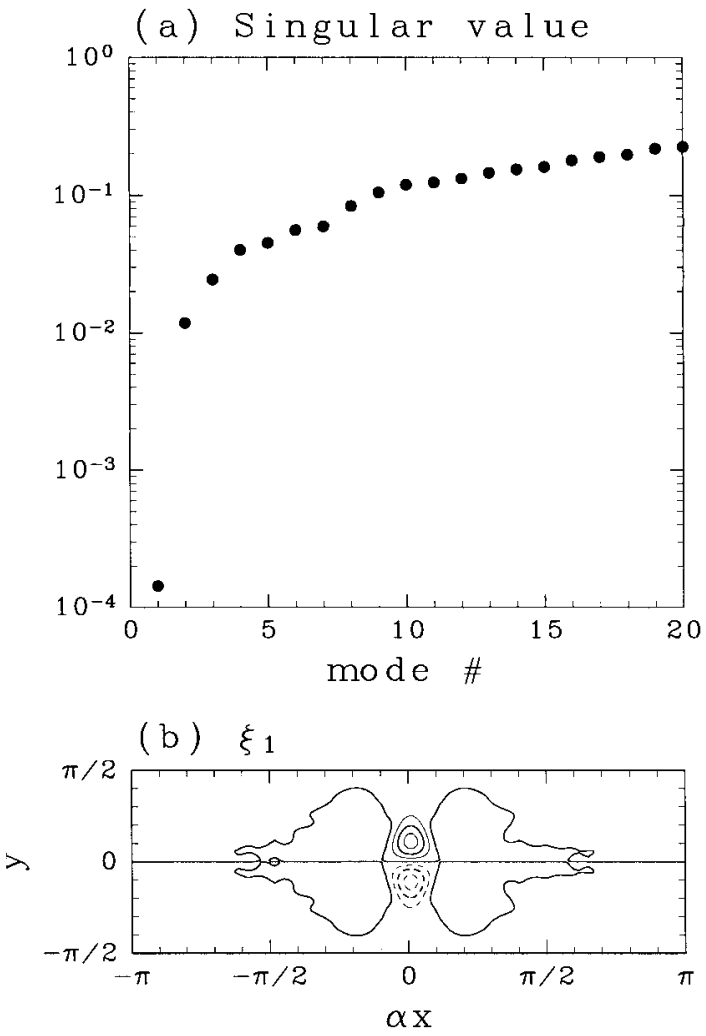

Fig. 5. SVD analysis for the blocking solution in the narrow channel model. (a) Distribution of the 20 smallest singular value $\sigma_{i}$. (b) Streamfunction field for the first right singular vector $\xi_{1}$. Contour interval is 1.0 and negative values are contoured by dashed lines.

\section{Wide channel model}

The streamfunction of the blocking solution in the narrow channel is noticeably deformed from the analytical modon solution, especially near the lateral boundaries. In order to reduce the influence of the boundaries to the results, we extend the channel width into twice the narrow channel width $\left(\pi L_{y}=21,000 \mathrm{~km}\right)$. We also increase the lateral truncation wave number $M$ in (8) from 21 to 42 so as to retain the same horizontal resolution as in the narrow channel.

Figure 6 shows the bifurcation diagram of the blocking solution and the zonal flow solution as in Fig. 1. The distortion of the streamfunction of the blocking solution (Fig. 7a) is rather reduced not only near the boundaries 


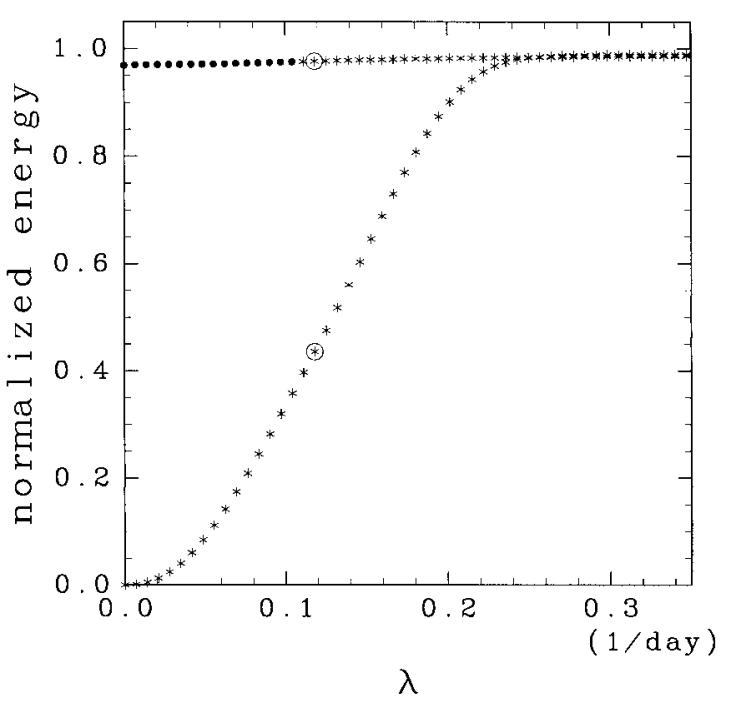

Fig. 6. As in Fig. 1 but for the wide channel model.
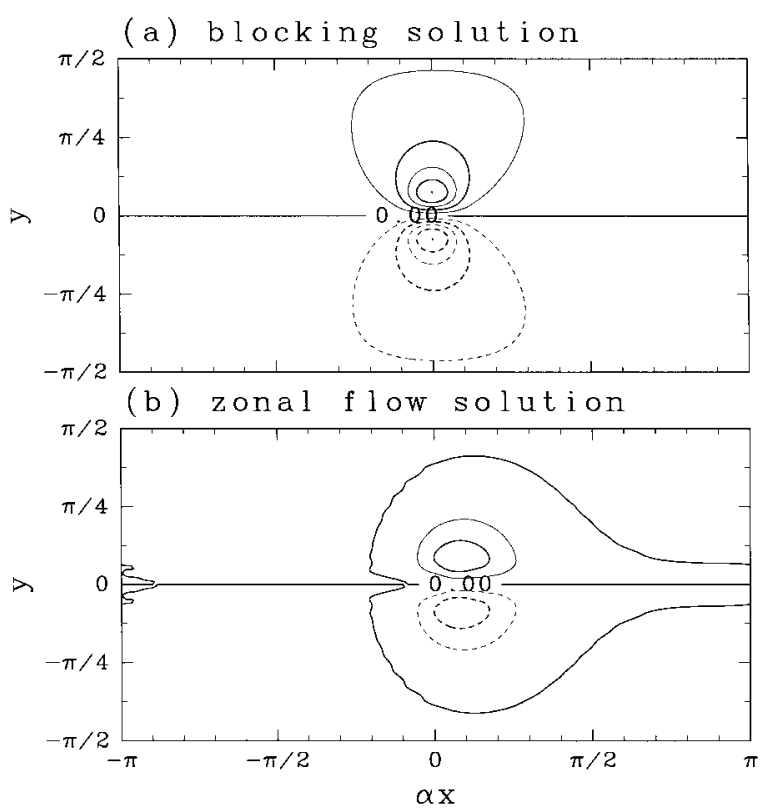

Fig. 7. Streamfunction $\psi_{0}$ of steady solutions at $\lambda=0.12$ day $^{-1}$ denoted by open circles in Fig. 6. Contour interval is 0.1 and negative values are contoured by dashed lines. (a) Blocking solution. (b) Zonal flow solution.

but also in the center of the channel: the amplitude of the dipole structure is slightly larger in the wide channel. Note that the contour interval in Fig. 7 is one half of that in Fig. 4 due

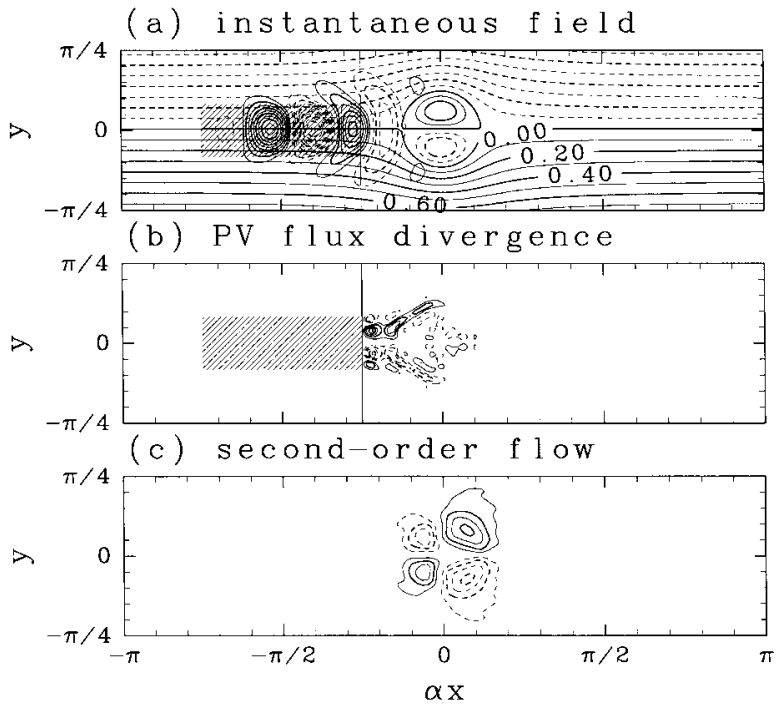

Fig. 8. As in Fig. 4 but for the blocking solution in the wide channel model. Only the half region of the model, $-\pi / 4<y<\pi / 4$ is shown. Contour intervals for (a), (b), (c) are $2.0 \times 10^{-5}$, $2.0 \times 10^{-6}, 2.0 \times 10^{-6}$.

to the nondimensionalization. The structure of the zonal flow solution (Fig. 7b) is noticeably unchanged. The linear stability property of both stationary solutions remains almost the same as that in the narrow channel: the blocking solution becomes unstable when $\lambda$ is smaller than 0.13 day $^{-1}$; the zonal flow solution is stable in the parameter range examined in this study. The analytical modon solution represented in the wide channel is also linearly unstable to infinitesimal perturbations. Thus, we could infer that the lateral boundaries in this channel model does not directly affect the stability property of the analytical modon solution. The growth rate and horizontal structure of the most unstable mode for the analytical modon solution are almost the same as those in the narrow channel.

Figure 8a shows an instantaneous streamfunction field of the perturbation $\psi_{1}$ at day 30 when the blocking solution is assumed as the basic flow. Since we focus upon the behavior in the center of the channel, only the central domain of $-\pi / 4<y<\pi / 4$ in the model will be shown in the following figures. The eddies are forced by the wavemaker $F_{1}$ of which region is denoted by the hatched rectangle in Fig. 8; the 

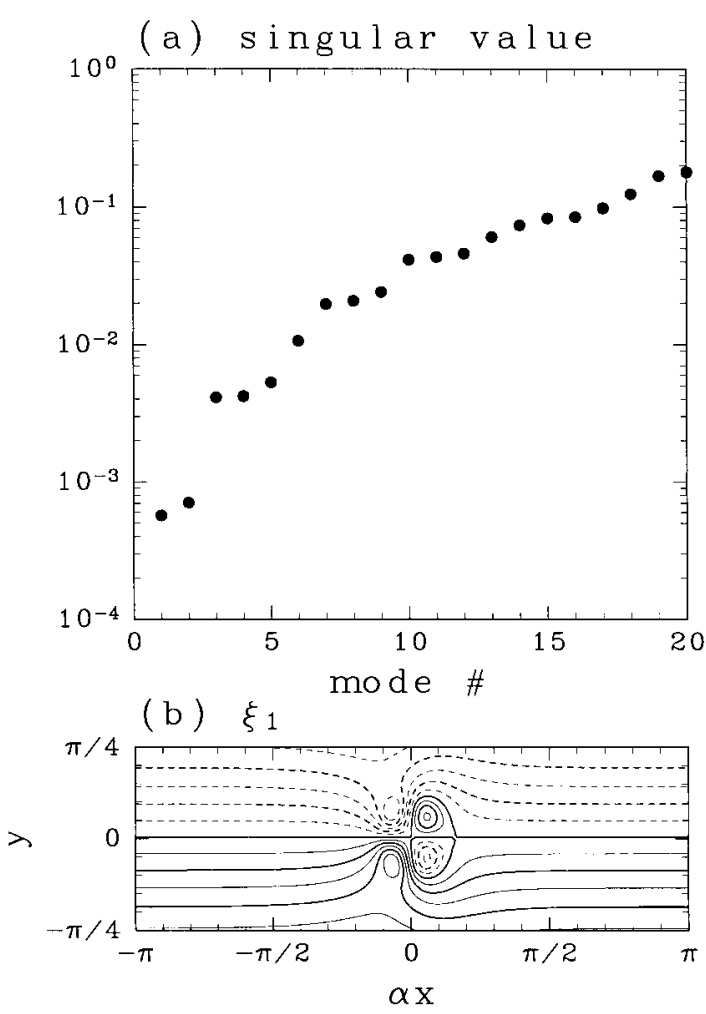

Fig. 9. As in Fig. 5 but for the blocking solution in the wide channel model. Contour interval for (b) is 0.2 .

wavemaker region has the same dimensional horizontal size as in the narrow channel model. The deformation of eddy structure and the horizontal distribution of the accompanied PV flux divergence upstream of the diffluence (Fig. 8b) are almost the same as those in the narrow channel model.

However, we find a remarkable difference in the second-order flow $\psi_{2}$ induced by the eddy forcing. In Fig. 8c, a quadruple structure is evident instead of the dipole structure in Fig. 4c. The downstream north-south vortex pair has a comparable magnitude to that of the upstream vortex pair. The polarity of these vortex pair is opposite to each other, and the second-order flow has a tendency to shift the basic blocking vortex pair downstream instead of enforcing them.

The difference in the response of the secondorder flow to almost the same PV flux divergence is also confirmed in the singular value decomposition analysis in Fig. 9a for the wide channel. The smallest singular value $\sigma_{1}$ is sev-

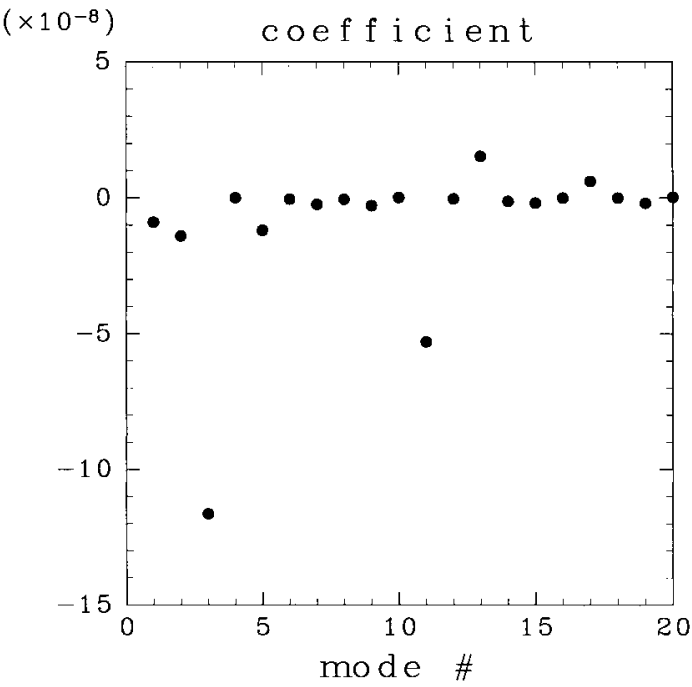

Fig. 10. Projection $\xi_{i}$ of the eddy PV convergence $\boldsymbol{f}$ onto each right singular vector $\left\langle\zeta_{i} \cdot \boldsymbol{f}\right\rangle / \sigma_{i}$ with the smallest 20 singular values $\sigma_{i}$ for the blocking solution in the wide channel.

eral times as large as that in the narrow channel, and its magnitude is comparable to the second smallest singular value. Although there is a weak quadrupole pattern in the streamfunction field of the first right singular vector $\xi_{1}$ (Fig. 9b), the zonal flow component which is absent in Fig. 8c dominates in its flow pattern. Moreover, the magnitude of the coefficient $\left\langle\zeta_{1} \cdot \boldsymbol{f}\right\rangle / \sigma_{1}$ in (22) associated with $\xi_{1}$ is not dominant as shown in Fig. 10a. Thus, the second-order flow $\overline{\psi_{2}}$ for the blocking solution in the wide channel has relatively strong sensitivity to the characteristic of the imposed eddy forcing. This implies that the resonant enhancement mechanism of the blocking solution by the transient eddies found in the narrow channel as well as in HM is due to the narrow meridional extent of the channel model. We will further examine the sensitivity of the secondorder flow to several eddy forcing characteristics in the next section.

Figure 11a shows an instantaneous perturbation streamfunction field at day 30 when the zonal flow solution (Fig. 7b) is assumed as the basic flow $\psi_{0}$. The perturbations induced by the same wavemaker $F_{1}$ (hatched region) as in Fig. 8 have rather complicated structure. Eddies in the center of the channel become fragmented 


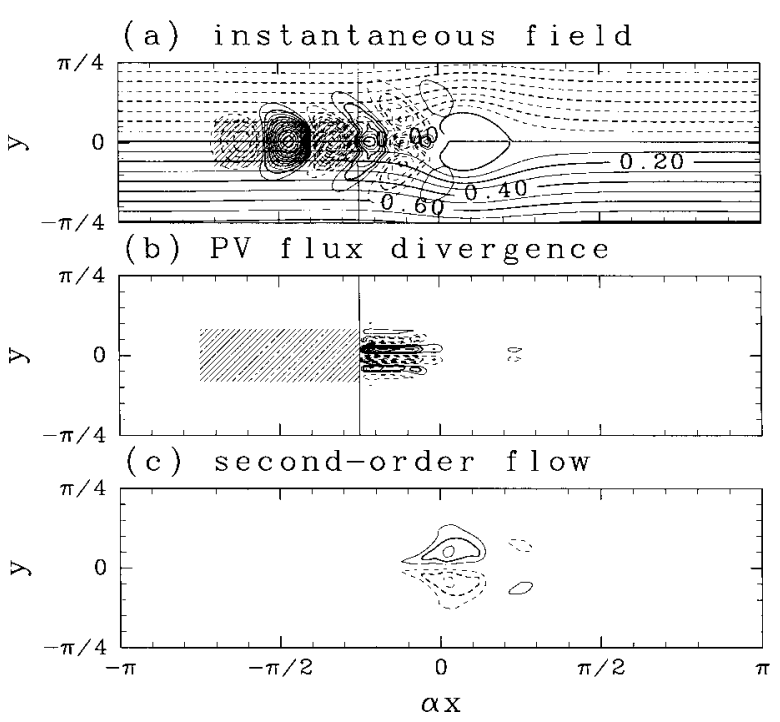

Fig. 11. As in Fig. 8 but for the zonal flow solution in the wide channel model.

associated with the stagnation region of the basic flow, and fade away due to the Ekman friction and the numerical diffusion. On the other hand, the meridionally split eddies due to weak diffluence of the basic flow survive further downstream compared with those for the blocking solution. Thus, the distribution of the timemean eddy PV flux divergence (Fig. 11b) is concentrated in the center of the channel and has a smaller meridional scale compared with that for the blocking solution (Fig. 8b). The second-order flow induced by the time-mean eddy PV flux divergence is shown in Fig. 11c. Because its horizontal pattern is similar to the zonal flow solution (Fig. 7b) and its polarity is also the same, it is suggested that the imposed perturbation has an effect to maintain the basic zonal flow solution. The singular value decomposition analysis of the zonal flow solution shows that the resonant enhancement mechanism of the basic flow is not efficient, and the second-induced flow crucially depends on the characteristic of the imposed wavemaker (not shown).

\section{Sensitivity of the second-order flow to the imposed eddies}

The results in the previous section suggest that the second-order induced flow is very sensitive to the characteristic of the imposed

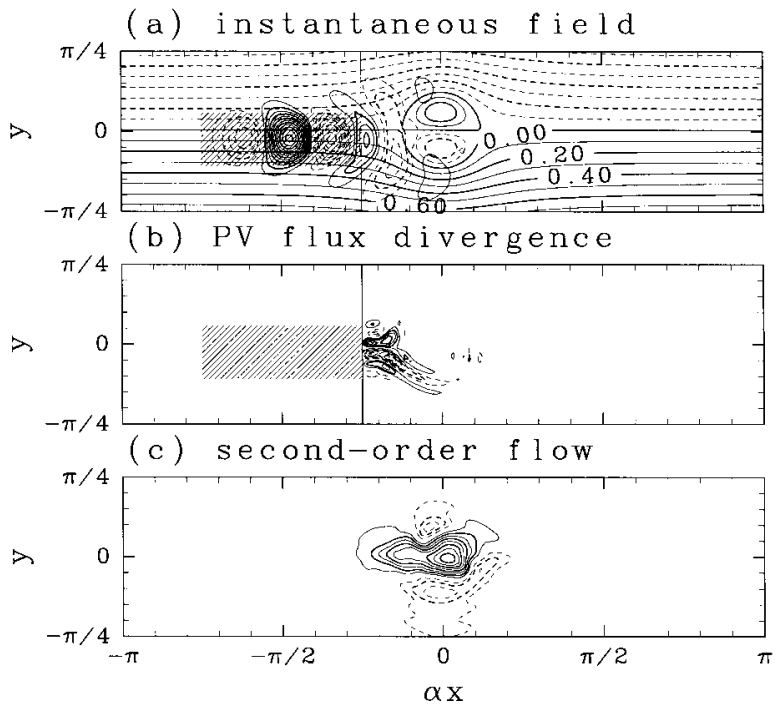

Fig. 12. Same as in Fig. 8 except that the wavemaker is shifted to the south by $15 \%$ of the modon radius $r_{0}$.

eddies. In order to illustrate this high sensitivity, we examine the dependence of the second-order flow on the meridional location and the horizontal size of the wavemaker. The blocking solution is assumed as the basic flow in this section.

\subsection{Meridional shift of the wavemaker}

Figure 12 shows a result when the wavemaker is shifted to the south by $15 \%$ of the modon radius $r_{0}$. The instantaneous streamfunction field of $\psi_{1}$ at day 30 (Fig. 12a) is almost identical to that in Fig. 8a except that eddies in the southern jetstreams retain larger amplitude compared with those in the northern jetstreams. The eddy PV flux divergence pattern (Fig. 12b) is also almost identical to that in Fig. 8b: a north/south, divergence/convergence dipole pattern resides upstream of the diffluence. On the other hand, the $\overline{\psi_{2}}$ flow pattern in Fig. $12 \mathrm{c}$ is drastically deformed in comparison with Fig. 8c: a dominant anti-cyclonic vortex is located near $y=0$ instead of the quadrupole pattern. This second-order flow will destroy the blocking dipole immediately since the antisymmetry with respect to $y=0$ in the blocking dipole is totally lost in Fig. 12c; the meridional shift of the wavemaker only by $5 \%$ of the modon radius is enough to extinguish the antisymmetry of the second-order flow. This is an 

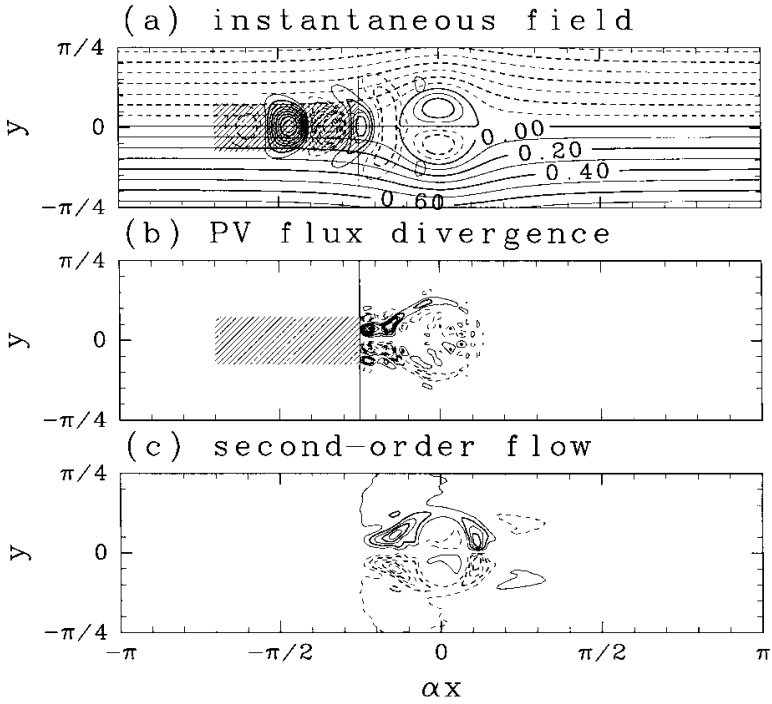

Fig. 13. Same as in Fig. 8 except that the zonal wavelength of the imposed eddies is $6,300 \mathrm{~km}$, and the eddy streamfunction at day 29 is drawn in (a).

example of high sensitivity of the second-order flow to the meridional shift of the wavemaker.

\subsection{Horizontal size of the wavemaker}

Next, the examine the sensitivity of the second-order flow to the horizontal scale of the imposed wavemaker $\Delta x$ and $\Delta y$ in (19) while the aspect ratio of the wavemaker is kept constant as in section 4 .

The further increase of the horizontal scale of the wavemaker does not give rise to qualitative change in the second-order flow; the secondorder flow retains a tendency to advect the blocking dipole downstream (not shown). On the other hand, the decrease of horizontal scale of the wavemaker brings about a drastic change in the second-order flow. Figure 13 and Fig. 14 show the results for the prescribed zonal wavelength of eddies are $6,300 \mathrm{~km}$ and $5,600 \mathrm{~km}$, respectively. The horizontal deformation of eddies associated with the blocking dipole in these two figures is apparently the same. The time-mean eddy PV flux divergence in Fig. 13b is also almost the same as that in Fig. 14b except that there is a slight enhancement of the north/south, divergence/convergence along the split jets around the blocking dipole in the former. However, the second-order flow is distinctively different between them: in Fig. 13c, a

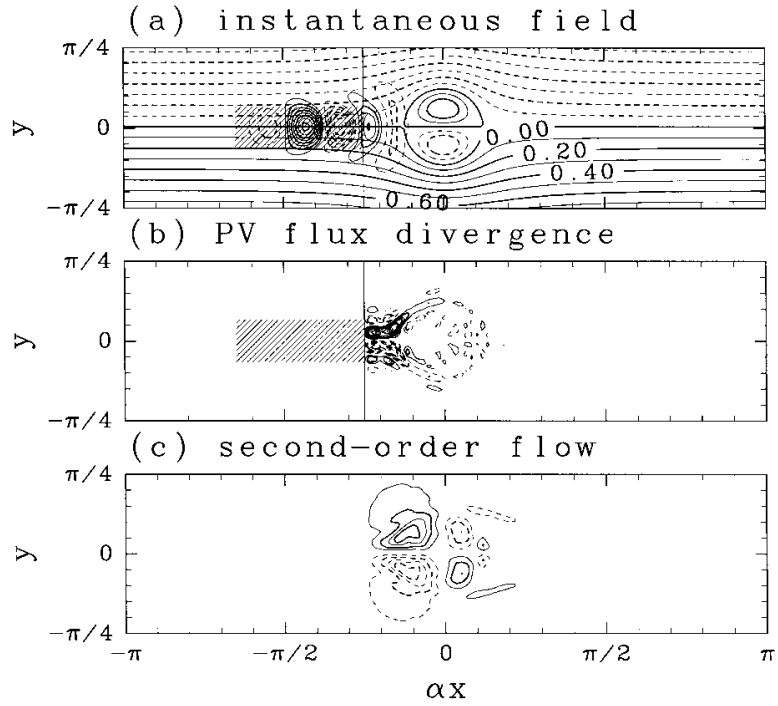

Fig. 14. Same as in Fig. 8 except that the wavelength of the eddies is $5,600 \mathrm{~km}$, and the eddy streamfunction at day 28 is drawn in (a).

weak dipole with the opposite polarity to the blocking dipole exists within the dipole of the basic flow while strong dipoles with the same polarity as the blocking resides outside the dipole of the basic flow. This pattern will tend to diffuse the vortex pair associated with the blocking; The flow in Fig. 14c has a quadrupole structure with the opposite polarity to that in Fig. 8c, and tends to shift the blocking dipole upstream.

These two experiments suggest that a small difference in the time-mean eddy PV flux divergence has a possibility to emerge a large difference in the second-order induced flow. Moreover, the effectiveness of the eddy straining mechanism is very sensitive to parameters prescribing the horizontal structure of migrating eddies.

\section{Concluding remarks}

In order to examine the effectiveness of the eddy straining mechanism on the maintenance of the blocking flow proposed by Shutts (1983), we investigate the mutual interaction between migrating eddies and stationary basic flows by using an equivalent barotropic $\beta$-channel model, which is all the same as in HM except that the channel width is enlarged twice to reduce the distortion of the flow field near the 
channel walls. The eddies which mimic the synoptic-scale waves are generated by an upstream wavemaker forcing. Their amplitude is assumed to be small enough so that the interaction is described by a quasi-linear framework as in HM. The model possesses two stationary solutions accompanying isolated structures in prescribed uniform westerlies when the vorticity forcing associated with the analytical modon solution is assumed: "blocking solution" which closely resembles the modon solution and "zonal flow solution" which is characterized by dominant zonal flows. The effectiveness of the eddy straining mechanism is assessed by the resemblance between the basic flow and the second-order flow induced by the time-averaged eddy potential vorticity (PV) divergence as in HM. The following results are obtained:

- When the basic flow is prescribed by linearly stable blocking solutions, the second-order flow induced by eddies has a quadruple structure, which tends to shift the blocking dipole downstream, instead of the dipole structure enforcing the blocking as shown in HM. On the other hand, the time-averaged eddy PV flux divergence has almost the same pattern as in HM: north/south, divergence/convergence dipole upstream of the diffluence of the basic flow.

- The superficial enhancement of the blocking dipole by the eddy straining mechanism indicated by HM is attributed to the false resonance characteristic of the basic flow due to the narrow channel model. This is also confirmed by the SVD analysis.

- The second-order flow for the linearly stable zonal flow solutions has a tendency to enforce the weak diffluence associated with the basic flow. The enhancement is not due to the resonant property of the basic flow but depends on the properties of imposed eddies.

- The second-order induced flow for the blocking solution is drastically deformed by a negligible distortion of the eddy PV flux divergence field due to a small change in the property of imposed eddies, such as their horizontal scale and the meridional position of the trains of eddies relative to the diffluent region.

Thus, our study revealed that the eddy straining mechanism for the maintenance of the blocking flow against the dissipation is not so

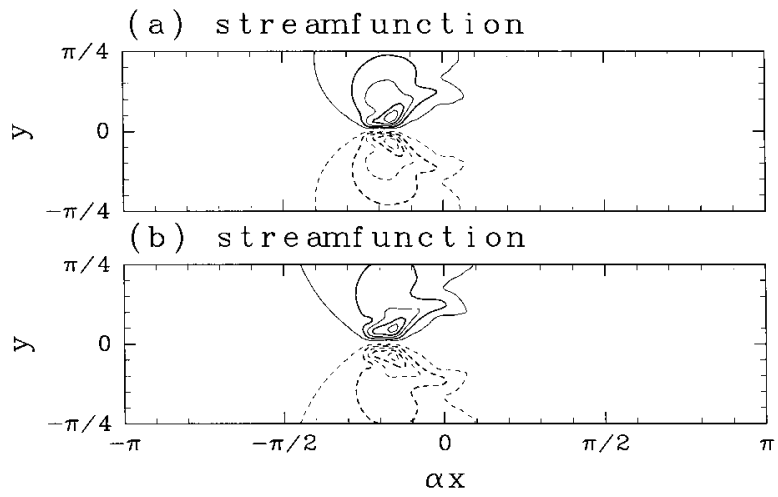

Fig. 15. Streamfunction tendency $-\nabla^{-2} \overline{J\left(\psi_{1}, q_{1}\right)}$ obtained from the eddy PV flux divergence of Fig. 13b for (a) and of Fig. 14b for (b), respectively. Contour intervals are $1.0 \times 10^{-8}$ and negative values are contoured by dashed lines. Zero contours are omitted.

effective as indicated in the previous studies, and its effectiveness is also very sensitive to the properties of migrating eddies as well as the assumed basic flow.

Furthermore, the high sensitivity of the second-order flow to a fine structure in the eddy PV flux divergence field will suggest that the downgradient eddy PV flux divergence structure upstream of the blocking flow does not necessarily imply the enforcement of the blocking flow by the synoptic eddies; observational studies (e.g., Shutts 1986; Mullen 1987) usually insist the enforcement only by indicating such a PV flux divergence field, or the corresponding geopotential tendency field simply obtained with an inverse Laplacian operator. In order to designate a large difference between the second-order flow and the tendency field from the PV flux divergence field, Fig. 15a and Fig. 15b show the streamfunction tendency fields $\partial \tilde{\psi} / \partial t$ obtained by $-\nabla^{-2} \overline{\boldsymbol{J}\left(\psi_{1}, q_{1}\right)}$ for the eddy PV flux divergence field in Fig. 13b and Fig. 14b, respectively. These two streamfunction fields are too similar to be distinguished while there is noticeable difference in the second-order flow as already shown. This difference suggests the importance of zonal asymmetry of the basic flow in computing the second-order flow. Moreover, the high sensitivity of the second-order flow to a fine structure of the eddy PV flux divergence implies that a data set with high spa- 
tial resolution is necessary to assess the role of the synoptic eddies on the maintenance of the blocking flow although a resultant second-order flow has a relatively large horizontal scale.

These results are obtained in the framework of a quasi-linear theory. Thus, it is necessary for the next study to conduct nonlinear experiments to confirm our results. The possibility to form the blocking flows only by the synoptic eddies from basic states with prevailing zonal flows suggested by Shutts (1983) has to be also examined for a future study in order to reveal the role of synoptic eddies in the dynamics of the blocking.

\section{Acknowledgments}

The authors would like to thank Profs. I. Hirota, B.J. Hoskins, H. Nakamura for their careful reading of and many constructive comments on the original manuscript. The comments from Prof. T.G. Shepherd and anonymous reviewers led to substantial improvements in the manuscript. The GFD-DENNOU library were used for the graphics. This study was supported by Grant-in-Aid for Scientific Research (C)(2)12640417 and (B)(2)11219203 from the Ministry of Education, Science, and Culture of Japan.

\section{References}

Haines, K. and J. Marshall, 1987: Eddy-forced coherent structures as a prototype of atmospheric blocking. Quart. J. Roy. Meteor. Soc., 113, 681-704.

Hoskins, B.J., I.N. James and G.H. White 1983: The shape, propagation and mean-flow interaction of large-scale weather systems. J. Atmos. Sci., 40, 1595-1612.
Itoh, H. and M. Kimoto, 1999: Weather regimes, lowfrequency oscillations, and principal patterns of variability: A perspective of extratropical low-frequency variability. J. Atmos. Sci., 56, 2684-2705.

Maeda, S., C. Kobayashi, K. Takano and T. Tsuyuki, 2000: Relationship between singular modes of blocking flow and high-frequency eddies. $J$. Meteor. Soc. Japan, 78, 631-645.

McWilliams, J.C., 1980: An application of equivalent modons to atmospheric blocking. Dyn. Atmos. Ocean, 5, 43-66.

, G.R. Flierl, V.D. Larichev and G.M. Reznik, 1981: Numerical studies of barotropic modons. Dyn. Atmos. Ocean, 5, 219-238.

Mukougawa, H., 1988: A dynamical model of "quasistationary" states in large-scale atmospheric motions. J. Atmos. Sci., 45, 2868-2888.

Mullen, S.L., 1987: Transient eddy forcing of blocking flows. J. Atmos. Sci., 44, 3-22.

Navarra, A., 1993: A new set of orthonormal modes for linearized meteorological problems. J. Atmos. Sci., 50, 2569-2583.

Nycander, J., 1992: Refutation of stability proofs for dipole vortices. Phys. Fluids. A, 4, 467-476.

Pierrehumbert, R.T. and P. Malguzzi, 1984: Forced coherent structures and local multiple equilibria in a barotropic atmosphere. J. Atmos. Sci., 41, 246-257.

Shutts, G.J., 1983: The propagation of eddies in diffluent jetstreams: eddy vorticity forcing of 'blocking' flow fields. Quart. J. Roy. Meteor. Soc., 109, 737-761.

_ 1986: A case study of eddies forcing during an Atlantic blocking episode. Adv. Geophys., 29, 199-226.

Yamagata, T., K. Sakamoto and M. Arai, 1990: Locally-induced nonlinear modes and multiple equiribria in planetary fluids. $P A G E O P H, \mathbf{1 3 3}$, 733-748. 